\title{
Spinal Surgery - Use of Dexmedetomidine for Myasthenia Gravis - A Case Report
}

\author{
Nighat Abbas', Syed Sajjad Alam*, Muhammad Rafique1, Sumayya Kausar ${ }^{1}$, Anees ur Rehman ${ }^{1}$ and Sarosh ul Hassan ${ }^{1}$ \\ ${ }^{1}$ Department of Anesthesiology, Critical Care and Pain Management, Liaquat National Hospital and Medical College, \\ Karachi, Pakistan
}

\begin{abstract}
Myasthenia gravis (MG) is a chronic autoimmune disease in which autoantibodies destroy acetylcholine receptors at motor end plat of neuromuscular junction which prevent skeletal muscle depolarization and contraction, causes muscle weakness and tiredness upon exertion with a tendency to be subsided after taking some rest or after taking anticholinesterase medication. Symptoms of progression of MG include the involvement of upper and lower extremities and muscle weakness that leads to inability in doing basic motor functions. The diagnosis of myasthenia gravis includes sign and symptoms, clinical examination and laboratory investigation of serum autoantibody (AChR autoantibodies) implicated in the disease pathology. Surgical procedures under general anesthesia in a patient with MG could be very challenging for an anesthetist because of pathophysiological manifestations of the disease. We report a case of a patient undergoing 4 level spinal fixation with coexistent myasthenia gravis (according to Osserman II B), requiring general anesthesia for spinal surgery in prone position. Myasthenia gravis affects the neuromuscular junction that is why it has a great significance of interest for the anesthetist.
\end{abstract}

Keywords: Myasthenia gravis, neuromuscular monitoring, dexmedetomidine.

\section{INTRODUCTION}

Myasthenia gravis affects the neuromuscular junction that's why it has a great significance of interest for the anesthetist. It is a chronic autoimmune disease in which auto antibodies destroy acetylcholine receptors at motor end plat of neuromuscular junction which prevent skeletal muscle depolarization and contraction, causes muscle weakness and tiredness upon exertion with tendency to be subsided after taking some rest or after taking anticholinesterase medication [1]. There are two clinical presentations of myasthenia gravis: ocular and generalized myasthenia. In ocular myasthenia, the weakness is limited to the eyelids and extra-ocular muscles whereas in generalized myasthenia, the weakness commonly affects ocular muscles, but it also involves a variable combination of bulbar, limb, and respiratory muscles [2].

In 1958, Osserman proposed placing patients with myasthenia gravis in five groups: I - localized (ocular), II - generalized (mild or moderate), III - acute fulminating, IV - late severe, and V - muscle atrophy. Later, Osserman divided group II into the sub classification: a) mild and b) moderate. Various modified criteria have been suggested over the years. This classification helps to grade the disease, assessment of perioperative risk and possible complications [3].

Symptoms of progression of MG includes the involvement of upper and lower extremities muscle weakness that

\footnotetext{
*Corresponding Author: Syed Sajjad Alam, Liaquat National Hospital and Medical College, Karachi, Pakistan; Email: sajjadalamdr15@gmail.com Received: September 25, 2019; Revised: March 18, 2020; Accepted: April 04, 2020 DOI: https://doi.org/10.37184/lnjpc.2707-3521.1.19
}

leads to inability in doing basic motor functions like raising the arms over the head and walking up and down the stairs. If the symptoms persist or left untreated can cause respiratory muscle weakness leads to respiratory failure because of airway muscles insufficiencies leading to reduced tidal volume and patient may need artificial ventilation, the condition called myasthenia crisis [4-6].

The diagnosis of myasthenia gravis includes sign and symptoms, clinical examination and laboratory investigation of serum autoantibody (AChR autoantibodies) implicated in the disease pathology [7]. Laboratory investigation of Serum acetylcholine receptors antibodies and Tensilon test confirm myasthenia gravis in almost 85 to $90 \%$ of cases [8,9]. On the basis of updated clinical trial, treatment of myasthenia gravis includes: a) symptomatic treatments facilitating neuromuscular transmission, (b) antibody-depleting treatments and (c) immunotherapeutic treatment strategies [10]. Some studies show that thymectomy plays an important role in reliving the symptoms of MG $[11,12]$.

Surgical procedures under general anesthesia in a patient with MG could be a very challenging for an anesthetist because of pathophysiological manifestations of the disease. So, before any surgical procedure under general anesthesia except emergency procedure, treatment overlying medical issues in these patients are necessary [13].

The prevention and anticipation of potential intraoperative or post-operative complication like myasthenia crisis and respiratory failure, needs special anesthetic considerations during perioperative period. 


\section{Case:}

This is a case of 55-year-old, $70 \mathrm{~kg}$ weighing female, known case of HTN and diagnosed case of myasthenia gravis classified in Osserman class Ilb during 2015 on list for 4 level fixation of spine and decompression due to severe backache radiating to right leg. Initially she noticed difficulty in swallowing for solids which became severe with time even for liquids in September 2015. She went to a local hospital where her initial workup was done which was unremarkable. Then autoantibody test was done which was positive for myasthenia gravis. Since then she had been on tab Amygra (Pyridostigmine) $300 \mathrm{mg}$ per day, few pain killers and multivitamins. She had been taking tab Candesartan $4 \mathrm{mg}$ per day and tab Carvedilol $6.25 \mathrm{mg}$ daily for hypertension since 15 years.

\section{Pre-anesthesia Assessment:}

Before the induction of anesthesia, pre-anaesthesia assessment was done. American Society of Anesthesiologists (ASA) standard vitals like blood pressure, pulse, oxygen saturation, ECG were recorded which were within normal limits. Then systemic examination like CNS, CVS, respiratory and renal examination was done which was found nonsignificant. Airway examination was thoroughly carried out which included thyromental distance and mallampati classification which anticipation is not difficult. Labs like complete blood count, urea/creatinine/electrolytes, PT, INR with all values were normal in range and chest $x$-ray was done to exclude the presence of chest infection. She had no history of ptosis, diplopia or dyspnea or extra-oral /intraoral swelling, edema or tenderness in preoperative assessment and consultation.

Our anesthetic plan included induction with propofol and maintenance with dexmedetomidine infusion and inhalation agent isoflurane. We planned to avoid neuromuscular blocking agents because of higher sensitivity of muscle relaxants in MG patients.

\section{Anesthetic Procedure:}

In operating room, standard monitoring as per ASA guidelines was done and intravenous access was obtained. Premedication was with $2 \mathrm{mg}$ midazolam and the loading dose of dexmedetomidine $70 \mathrm{mcg}(1 \mathrm{mcg} /$ $\mathrm{kg}$ ) in $100 \mathrm{ml}$ normal saline over 10 mins to be followed by a maintenance infusion of $0.6 \mathrm{mcg} / \mathrm{kg} /$ hour, then propofol at $2 \mathrm{mg} / \mathrm{kg}$ was given and the patient was intubated during propofol apnea less than 15 seconds. The Intubation was found uncomplicated. Therefore invasive arterial monitoring was done. Maintenance of anesthesia was achieved with isoflurane 0.8 to $1 \mathrm{MAC}$ and dexmedetomidine infusion was titrated to achieve desired clinical effects with the dose ranging from 0.2 to $1 \mathrm{mcg} / \mathrm{kg} / \mathrm{hour}$. Nalbuphine $0.1 \mathrm{mg} / \mathrm{kg}$ was given at surgical incision. During anesthesia, the patient's mechanical ventilation parameters were adjusted on the basis of standard anesthesiological evaluation. Doses of all anesthetic agents were modified according to the clinical assessment of the depth of anesthesia. 4 level lumber spine from L1 to L4 fixation and decompression was done in prone position in the period of 3 hours. Patient was remained hemodynamically stable throughout the surgery. At the end of surgery patient was placed on supine position. Isoflurane and infusion of dexmedetomidine were discontinued. Soon after 5 to 8 mins, patient opened her eyes and she was extubated smoothly without cough after the return of adequate muscle power and respiratory efforts. Patient was shifted in post anesthesia care unit (PACU) where she was again monitored and $100 \%$ oxygen supplementation was given. She was kept in PACU for an hour and was hemodynamically stable throughout and maintaining oxygen saturation during PACU stay. She had no complain of nausea and vomiting. After having all clinical examination of muscle tone and power, patient was shifted in ward.

\section{DISCUSSION}

There are risk of severe complications and even death associated with surgery and anesthesia in patients with myasthenia gravis due to higher sensitivity to muscle relaxants [14]. That is why these patients may often require prolonged ventilation in the ICU $[15,16]$. So, an anesthetist should avoid the use of muscle relaxant agent. However in some surgeries like abdominal surgery, use of muscle relaxants are necessary to improve operating conditions. For this purpose many anesthesiologist use short or intermediate acting non depolarizing muscle relaxants of 1/10th dose [17].

Myasthenia gravis is classified by Osserman into five different subgroups that increase in degree of severity: i.e. Group I which includes localized ocular symptoms, Group IIA and IIB which include mild to moderate generalized muscle weakness, Group III which includes severe generalized myasthenia gravis with characteristics such as fulminant hepatic failure with or without respiratory complications, Group IV which includes complete muscular atrophy with resulting myasthenia crisis due to respiratory failure [18]. This classification is very helpful in clinical management of myasthenia gravis patients.

Dexmedetomidine is an alpha2 agonist sedativeanalgesic. It inhibits norepinephrine release. Locus coeruleus is its effector site for sedative action and spinal cord as effector site for analgesic action. That is why dexmedetomidine reduces the opioid consumption and minimum alveolar concentration of inhalational agents [19]. As dexmedetomidine reduces the opioid consumption, the incidence of post-operative nausea and vomiting also reduces [20]. The cardio protective effect of dexmedetomidine is to reduce sympathetic response to surgical stimulation and reduce oxygen consumption [16]. Dexmedetomidine has anti shivering effects [21]. Shivering can worsen pain post-operatively, increase oxygen consumption which can be harmful for 
the elderly patients. Shivering can cause increase in carbon dioxide production, lactic acidosis and intraocular pressure [22]. Most of the advantages as discussed above were also observed in our patient. Intraoperative inhalation dose of isoflurane (MAC) were also reduced. Our patient was extubated after the surgery in supine position with no residual respiratory depression and no postoperative pain or shivering.

Dexmedetomidine shows biphasic blood pressure response. It increases first in transient blood pressure then followed by decrease in blood pressure due to its sympatholytic action. This action of dexmedetomidine causes baroreflex mediated decrease in heart rate, centrally mediated decrease in sympathetic tone and increase in vagal tone [16]. As we administered the loading dose of dexmedetomidine over 10 mins we found no change in blood pressure and heart rate and the patient was hemodynamically stable throughout the procedure.

\section{CONCLUSION}

Dexmedetomidine was found as a good sedative drug with analgesic effects. The opioid consumption and minimum alveolar concentration of inhalational agents are also reduced when using with dexmedetomidine. This drug can safely be used in patients with myasthenia gravis undergoing spinal surgery, keeping the patient deeply sedate, pain free and relax but one should always monitor hemodynamic changes and prepare vagolytic and symphathomimitic drugs before using dexmedetomidine.

\section{CONFLICT OF INTEREST}

All authors declare no conflict of interest.

\section{ACKNOWLEDGEMENTS}

Declared none.

\section{REFERENCES}

1. Clinical overview of MG. Myasthenia Gravis Foundation of America. Available at: http://www.myasthenia.org/HealthProfessionals/ ClinicalOverviewofMG.aspx (Accessed on: April 1, 2019).

2. Myasthenia gravis fact sheet. National Institute of Neurological Disorders and Stroke. Available at: http://www.ninds.nih.gov/ disorders/myasthenia_gravis/detail_myasthenia_gravis.htm (Accessed on: April 1, 2019).

3. Mincewicz G, Aloszko A, Nyka W, Bilińska M, Krzykowski G, Nyka WM. The comparison of selected spirometric parameters with functional muscles exercise evaluation in patients with myasthenia. Pol Merkur Lek 2007; 22: 177-81.
4. Keesey JC. Clinical evaluation and management of myasthenia gravis. Muscle Nerve 2004; 29: 484-505.

5. Bird SJ. Overview of the treatment of myasthenia gravis. Available at: https://www.uptodate.com/contents/search (Accessed on: April 1, 2019).

6. Wendell LC, Levine JM. Myasthenic crisis. Neurohospitalist 2011; 1: 16-22.

7. Gilhus NE. Myasthenia gravis. N Engl J Med 2016; 375: 2570-81.

8. Drachman DB. Myasthenia gravis. N Engl J Med 1994; 330: $1797-$ 810.

9. Daroff RB. The office tensilon test for ocular myasthenia gravis. Arch Neurol 1986; 43: 843-4.

10. Melzer N, Ruck T, Fuhr P, Gold R, Hohlfeld R, Marx A, et al. Clinical features, pathogenesis, and treatment of myasthenia gravis: a supplement to the guidelines of the German Neurological Society. J Neurol 2016; 263: 1473-94.

11. Wolfe GI, Kaminski HJ, Aban IB, Minisman G, Kuo H-C, Marx A, et al. Randomized trial of thymectomy in myasthenia gravis. $\mathrm{N}$ Engl J Med 2016; 375(6): 511-22.

12. Bird SJ. Role of thymectomy in patients with myasthenia gravis. Available at: https://www.uptodate.com/contents/search (Accessed on: April 4, 2019).

13. Jamal BT, Herb K. Perioperative management of patients with myasthenia gravis: prevention, recognition, and treatment. Oral Surg Oral Med Oral Pathol Oral Radiol Endod 2009; 107: 612-5.

14. Dillon FX. Anesthesia issues in the perioperative management of myasthenia gravis. Semin Neurol 2004; 24: 83-94.

15. Haroun-Bizri S, Maalouli J, Deeb P, Baraka A. Anesthetic management for a patient with myasthenia gravis undergoing coronary artery bypass graft. Middle East J Anesthesiol 2003; 17 : 299-305.

16. Heliopoulos I, Patlakas G, Vadikolias K, Artemis N, Kleopa KA, Maltezos $\mathrm{E}$, et al. Maximal voluntary ventilation in myasthenia gravis. Muscle Nerve 2003; 27: 715-9.

17. Bom A, Bradley M, Cameron K, Clark JK, Egmond JV, Feilden H, et al. A novel concept of reversing neuromuscular block: chemical encapsulation of rocuronium bromide by a cyclodextrin-based synthetic host. Angew Chem Int Ed Engl 2002; 41: 266-70.

18. Osserman K. Myasthenia Gravis. New York: Grune \& Stratton 1958, pp. 78-80.

19. Reel B, Maani CV. Dexmedetomidine. StatPearls: Treasure Island (FL): StatPearls Publishing 2020.

20. Lee HS, Yoon HY, Jin HJ, Hwang SH. Can dexmedetomidine influence recovery profiles from general anesthesia in nasal surgery? Otolaryngol Head Neck Surg 2018; 158: 43-53.

21. ZhangJ, ZhangX, Wang H, Zhou H, Tian T, Wu A. Dexmedetomidine as a neuraxial adjuvant for prevention of perioperative shivering: meta-analysis of randomized controlled trials. PLoS ONE 2017; 12(8): e0183154.

22. Elvan E, Oc B, Uzun S, Karabulut E, Coşkun F, Aypar U. et al. Dexmedetomidine and postoperative shivering in patients undergoing elective abdominal hysterectomy. Eur J Anaesth 2008; 25: 357-64. 